News

\title{
Nutraceuticals: what are they and what do you do with them?
}

\section{News}

Nutraceuticals have been around since mankind started to consume food. What they are and how they have been used were just not recognized as such - since the term is a relatively new invention. Likewise, using the term nutraceuticals in relation to dairy animals is only recently becoming part of the vocabulary for dairy producers, even though they may have been using them all along.

Nutraceuticals can be defined as "a food (or part of a food) that provides medical or health benefits." The term comes from combining the words "nutrition" and "pharmaceutical." However, the word nutraceutical is really a broad umbrella term to describe a variety of products typically derived from food sources. Nutraceuticals can be classified in different ways based on origin, such as from plants (like tomatoes), animals (like fish oil), microbes (like the probiotic Lactobacillus) or minerals (like calcium). They also can be classified by health issues such as arthritis (like glucosamine). The nutraceuticals used for animals are the same basic ones as used for humans: vitamins, minerals, amino acids, essential fatty acids, antioxidants, probiotics, enzymes and herbs. Indeed, the use of many nutraceutical ingredients for animals in supplements or feeds initially stemmed from human use and is now being applied for animal use. Let's look at some of the more common nutraceuticals in dairy cow rations and supplements. These can be considered as several classes of compounds. The following is a short list:

\section{Direct-fed microbials}

The terms direct-fed microbials and probiotics are used interchangeably. Direct-fed microbials (or probiotics) refer to living organisms (bacteria, yeast) and should not be confused with prebiotics, compounds that promote the growth of gut bacteria (e.g., yeast culture, oligosaccharides) but are not living organisms. It is believed that gut bacteria have requirements for specific nutrients that may not be adequately provided by the animal's diet. Feeding these nutrients may promote the growth of gut bacteria, thereby improving the microbial profile in the gut. Some direct-fed microbial products contain viable yeast and other fungi (like Saccharomyces cerevisiae or Aspergillus oryzae) in addition to bacteria.

\section{Enzymes}

Fibrolytic (fiber-digesting) enzymes have been tested as forage additives for dairy diets with variable but encouraging results. In a recent trial conducted at the University of Idaho, mid-lactation cows were fed an alfalfa hay and silage-based total mixed ration (TMR), where the forage was sprayed with an enzyme preparation 24hours before feeding. Daily production by the cows' enzyme-treated forage increased by $1.3 \mathrm{~kg}$ of milk, $0.8 \mathrm{~kg}$ of fat and $0.6 \mathrm{~kg}$ of protein. Commercial enzyme products marketed as feed supplements are bacterial or fungal fermentation extracts. These preparations vary widely in the types and concentrations of specific enzymes they contain. As a result, their ability to digest particular feeds is also variable. Most are marketed on the basis of their ability to break down fiber, but many also digest other substrates, such as starch and protein.
Volume 2 Issue I - 2015

Tom Whitten
Technical Field Support Veterinarian, Vets Plus, USA

Correspondence: Tom Whitten, DVM, Technical Field Support, Vets Plus, Inc., Glenwood City,Wisconsin, USA, Tel 7I5 78I 980I,Email doctorwhitten@gmail.com

Received: November 04, 2014 | Published: January 29, 2015

This variation in activity suggests the potential to select particular enzymes for application to specific feeds.

\section{Botanicals and herbs}

Extracts of cinnamon, cloves and pepper have been standardized for use in dairy rations. These compounds have demonstrated the ability to change rumen fermentation patterns to favor more efficient feed utilization. The use of botanical extracts and herbs is an exciting future area that will continue to see new uses of these well-known human herbs and ingredients for dairy applications.

\section{Antioxidants}

Vitamin E and beta-carotene have received much attention in the last few decades due to their ability to scavenge damaging free radicals at the cellular level. Beta-carotene has been best studied since in most countries it is the most common carotenoid in fruits and vegetables. However, in the U.S., lycopene from tomatoes now is consumed in approximately the same amount as beta-carotene. Antioxidants (including carotenoids) have been studied for their ability to prevent chronic disease. Beta-carotene and other carotenoids have antioxidant properties in vitro and in animal models.

Mixtures of carotenoids or associations with other antioxidants (e.g. vitamin E) can increase their activity against free radicals. The usefulness of Vitamin $\mathrm{E}$ in immune response and mastitis prevention has been studied extensively. Nutritionists often formulate rations with higher Vitamin E levels for cows around calving time.

\section{Fats}

Very few nutraceuticals have received as much attention in recent years as the feeding of specific fats to dairy cows. Feeding a fat supplement enriched in conjugated linoleic acid (CLA) decreases milk fat and enhances energy balance in heat-stressed lactating dairy cows. There have been several commercial products developed using specific fats from fish oil and palm oil that have demonstrated an effect on reproductive performance, production and mitigation of heat stress. Fats are long chains of carbon with hydrogen atoms, and the naming and structure is complicated unless one has an organic 
chemistry background. There has been much discussion of the health benefits in animals and people of the Omega- 3 fatty acids and the Omega- 6 fatty acids.

Omega- 3 fatty acids are fats commonly found in marine and plant oils. They are polyunsaturated fatty acids with a double bonded carbon atom $(\mathrm{C}=\mathrm{C})$ starting after the third carbon atom from the end of the carbon chain. The fatty acids have two ends-the acid $(\mathrm{COOH})$ end and the methyl (CH3) end. The location of the first double bond is counted from the methyl end, which is also known as the omega $(\omega)$ end or the $n$ end. Conversely, Omega- 6 fatty acids are a family of unsaturated fatty acids that have in common a final carbon-carbon double bond in the n- 6 position - that is, the sixth bond, counting from the methyl end (CH3).

\section{Selenium as a nutraceutical}

Feeding organic selenium as selenium yeast for the periparturient period during hot summer months increased plasma selenium concentrations, improved both neutrophil function and immuno- responsiveness, reduced occurrence of fevers, improved uterine health and increased second-service pregnancy rate. Responses differed between primiparous and multiparous cows.

As can be seen from this short discussion, nutraceuticals have been used by dairy producers all along, even though they probably were not aware that the supplements or feed ingredients they were giving their animals were considered nutraceuticals. The use of nutraceuticals continues to grow for the human market. Likewise, the use of nutraceuticals will continue to grow on the animal market as new ideas for old uses of human nutraceuticals is applied to the dairy industry.

\section{Acknowledgements}

None.

\section{Conflict of interest}

Author declares that there is no conflict of interest. 